\title{
A set of systematic reviews to help reduce inappropriate prescribing to older people: study protocol
}

Yolanda V. Martinez ${ }^{1 \dagger}$, Anna Renom-Guiteras ${ }^{2,3 \dagger}$, David Reeves ${ }^{1 *}$, R. Erandie Ediriweera de Silva ${ }^{1,4}$, Aneez Esmail ${ }^{1}$, Ilkka Kunnamo ${ }^{5}$, Anja Rieckert ${ }^{2}$, Christina Sommerauer ${ }^{2}$ and Andreas Sönnichsen ${ }^{2}$

\begin{abstract}
Background: Multimorbidity and polypharmacy are common in older people. Assessment tools or lists of criteria aimed at supporting prescription decisions for older people exist, but have often been based on expert opinion with insufficient consideration of the evidence available. The present paper describes the methods we are using to systematically review the existing evidence on the efficacy and safety of the most commonly prescribed drugs for older people in the management of their chronic medical conditions and to develop recommendations to reduce inappropriate prescriptions for incorporation into the Comprehensive Medication Review (CMR) tool developed by the PRIMA-eDS European project.
\end{abstract}

Methods: We selected the 20 most relevant drugs/drug classes in terms of prescription volumes and risk of hospitalisation for older people and the most relevant indications for the most common chronic conditions among older people and a total of 35 distinct drug-indication pairs were chosen. Based on clinical considerations we collapsed some indications together, reducing the 35 pairs to a final total of 22 separate systematic reviews (SR). A common methodology will be used for each individual SR, based on the methodological manuals of the Cochrane collaboration and the PRISMA statement for reporting systematic reviews. Our search strategy will have a staged approach where we initially search for systematic reviews and meta-analyses, but if relevant reviews are not found, then search for individual studies (controlled intervention and observational studies). Our pilot work and initial scoping of the literature suggested that very few, relevant individual trials or existing systematic reviews have researched or reported exclusively on older people. Therefore, sufficient data might not be available to perform meta-analysis but we will provide a narrative synthesis describing characteristics and findings of included studies. The collected evidence will be used to construct recommendations on when not to use or to discontinue a drug, or when to reduce its dose. Recommendations will be developed in team meetings using the GRADE methodology to reflect the strength of the recommendation and the quality of the evidence. Recommendations will be built into the CMR tool.

Discussion: This protocol has been prepared for a series of systematic reviews which will provide research-based evidence to develop recommendations to reduce inappropriate polypharmacy in older people as part of the CMR tool of the PRIMA-eDS project.

Keywords: Polypharmacy, Multimorbidity, Older adults, Inappropriate prescribing

\footnotetext{
* Correspondence: david.reeves@manchester.ac.uk

${ }^{\dagger}$ Equal contributors

${ }^{1} \mathrm{NIHR}$ School for Primary Care Research, Manchester Academic Health

Science Centre, University of Manchester, Manchester, England, UK

Full list of author information is available at the end of the article
} 


\section{Background}

As a consequence of increasing life-expectancy and medical progress, multimorbidity and its corollary polypharmacy have been increasing in recent years and this is seen most distinctly in older adults [1,2]. Multimorbidity defined as two or more chronic conditions affects more than half of the population $>75$ years of age $[3,4]$. Depending on setting between $34 \%$ and $59 \%$ of people $>75$ years are exposed to five or more drugs [5-7]. A study of people attending the emergency department at a hospital in London reported that half of the people over 75 years of age were on five or more prescription drugs [5], and another study found that $24 \%$ of residents admitted to 57 nursing homes in 8 European countries were prescribed ten or more drugs [8].

The volume of good quality clinical trial evidence for the use of medications in older people is limited as this population group has been often excluded $[9,10]$. Thus, although the use of many drugs may be recommended by clinical guidelines, guidelines themselves are often based on evidence from younger populations and older people may not obtain the same benefits [11]. Many of the drugs often prescribed to older people with comorbidity have an important risk of adverse events and hospitalisation in this population $[1,12,13]$. There is therefore a necessity to carefully weigh the risks and benefits of medication in older multimorbid people [14].

Several authors have developed assessment tools or lists of criteria aimed at supporting prescription decisions for older people [15]. However, these assessment tools have often been principally based on expert opinion with insufficient consideration of the evidence available. Some authors have reviewed and narratively synthetized the evidence available on the use of certain drugs with older people. However, to the best of our knowledge no study has systematically reviewed and analysed the benefits and risks of the drugs most commonly used by older people.

PRIMA-eDS is a European Union 7th Framework Programme project (grant agreement No. 305388) across 5 European countries (Austria, Finland, Germany, United Kingdom, Italy) whose main objective is to develop and trial an electronic decision support tool (Comprehensive Medication Review tool [CMR tool]) to reduce polypharmacy in older people with multiple chronic conditions. The present paper describes the methods we are using to systematically review the existing evidence on the efficacy and safety of the most commonly prescribed drugs for older people in the management of their chronic medical conditions and to develop recommendations to reduce inappropriate prescriptions for incorporation into the CMR tool.

\section{Methods}

\section{Selection of drug classes}

From prescribing data taken from national reports and health insurance companies for three of the PRIMA-eDS participating countries (Social Insurance Institute of Finland/Statistical Database Kelasto in Finland (Jaana Harsia-Alatalo, People aged over 75 using prescription drugs, Social Insurance Institute of Finland, personal communication, 2012); Medicines Utilisation Monitoring Centre (OsMed) in Italy [16], research network of the Italian General Practice (Giuliano Piccoliori, The most common drugs in people over 74, personal communication, 2012); and AOK North Rhine-Westphalia in Germany (AOK Northwest Department of Pharmacology, List of most commonly prescribed drugs in people $>65$ years of age, Dortmund, 2012, unpublished data)), and from a pilot study carried out in Austria [17], we identified seventy five drugs or drug classes as the most commonly prescribed to people aged 65 years and older. In conjunction with this, we conducted a literature search for information about which drugs are most often responsible for hospitalisation due to adverse events in the general population and in older people $[12,18-21]$. From this we selected the 20 most relevant drugs or drug classes in terms of prescription volumes and risk of hospitalisation. Some medications can be used for more than one indication and in these cases we included the most relevant indications, giving priority to the most common chronic conditions among older people, i.e. cardiovascular disease (including coronary heart disease, cerebrovascular disease and peripheral vascular disease), heart failure, hypertension, atrial fibrillation, type 2 diabetes mellitus, musculoskeletal disorders including pain, COPD, and mental diseases [22]. We identified a total of 35 distinct drug-indication pairs (Table 1).

\section{The systematic review methodology}

A separate systematic review (SR) will be conducted for each drug-indication pair. Based on clinical considerations we collapsed some indications together, reducing the 35 pairs to a final total of 22 SRs (Table 1). Each review aims to assess the efficacy and safety of the use of that particular drug with older people in the management of the associated indication or indications. A common methodology will be used for each individual SR, based on the methodological manuals of the Cochrane collaboration [23] and the PRISMA statement for reporting systematic reviews [24].

\section{Preparatory phase}

We developed a Protocol Template (PT) and a Standard Operating Procedures (SOP) document specifying the methodology for the SRs in detail, for reviewers to follow and to achieve uniformity of approach (the PT and SOP can be seen in Additional files 1 and 2). To refine our methodology, the PT and SOP were piloted by two researchers (YVM and ARG) who undertook a SR of the efficacy and safety of metformin in the care of older people with type 2 diabetes. 
Table 1 List of drugs and drug classes covered, corresponding indications and SRs performed

\begin{tabular}{|c|c|}
\hline Drug or drug class & Indication \\
\hline \multirow[t]{3}{*}{ Proton pump inhibitors } & GERD/GORD (SR-1) \\
\hline & $\begin{array}{l}\text { Gastrointestinal ulcer } \\
\text { (gastric, duodenal) (SR-1) }\end{array}$ \\
\hline & Dyspepsia (SR-1) \\
\hline $\begin{array}{l}\text { Anti- inflammatory and } \\
\text { anti- rheumatic products, } \\
\text { non-steroids (NSAIDs) }\end{array}$ & Musculoskeletal disorders (SR-2) \\
\hline Opioids & Pain (SR-3) \\
\hline $\begin{array}{l}\text { HMG CoA reductase } \\
\text { inhibitors (Statins) }\end{array}$ & $\begin{array}{l}\text { Prevention of cardiovascular } \\
\text { disease (SR-4) }\end{array}$ \\
\hline \multirow[t]{2}{*}{ Beta blocking agents } & Hypertension (SR-5) \\
\hline & Heart failure (SR-6) \\
\hline $\begin{array}{l}\text { Angiotensin Converting } \\
\text { Enzyme inhibitors (ACE-I) }\end{array}$ & Hypertension (SR-7) \\
\hline \multirow{3}{*}{$\begin{array}{l}\text { Low-ceiling diuretics, Thiazides } \\
\text { and Sulfonamides }\end{array}$} & Heart failure (SR-8) \\
\hline & Oedema (SR-8) \\
\hline & Hypertension (SR-9) \\
\hline \multirow[t]{2}{*}{ Calcium Channel Blockers (CCBs) } & Angina (SR-10) \\
\hline & Hypertension (SR-11) \\
\hline Nitrates & Angina (SR-12) \\
\hline \multirow[t]{5}{*}{ High ceiling diuretics } & Heart failure (SR-13) \\
\hline & Oedema (SR-13) \\
\hline & Hypertension (SR-13) \\
\hline & Liver failure (SR-13) \\
\hline & Renal failure (SR-13) \\
\hline Platelet aggregation inhibitors & $\begin{array}{l}\text { Prevention and treatment of } \\
\text { cardiovascular disease including } \\
\text { cerebral infarction and transient } \\
\text { ischaemic attack, coronary disease } \\
\text { and peripheral artery occlusive } \\
\text { disease (SR-14) }\end{array}$ \\
\hline \multirow{2}{*}{$\begin{array}{l}\text { Vitamin K antagonists (VKA) } \\
\text { Direct thrombin inhibitors and } \\
\text { Direct factor Xa inhibitors (New } \\
\text { Oral Anticoagulants (NOACs)) }\end{array}$} & $\begin{array}{l}\text { Deep vein thrombosis/lung } \\
\text { embolism (SR-15) }\end{array}$ \\
\hline & $\begin{array}{l}\text { Thromboembolism in atrial } \\
\text { fibrillation (SR-16) }\end{array}$ \\
\hline \multirow[t]{2}{*}{ Digitalis glycosides } & Heart failure (SR-17) \\
\hline & Atrial fibrillation (SR-17) \\
\hline Metformin & Type 2 diabetes (SR-18) \\
\hline $\begin{array}{l}\text { Dipeptidyl peptidase } 4 \text { (DPP-4) } \\
\text { inhibitors (Gliptins) }\end{array}$ & Type 2 diabetes (SR-19) \\
\hline Pioglitazone & Type 2 diabetes (SR-20) \\
\hline Sulfonylureas & Type 2 diabetes (SR-21) \\
\hline Glinides & Type 2 diabetes (SR-21) \\
\hline Insulin and analogues & Type 2 diabetes (SR-22) \\
\hline
\end{tabular}

GERD gastroesophageal reflux disease, GORD gastro-oesophageal reflux disease, HMG COA 3-hydroxy-3-methylglutaryl-coenzyme A, Direct factor Xa inhibitors xabans
Next, a team of reviewers competent in English was established and trained on the methods and standard operating procedures by means of 7 workshops delivered by three researchers (YVM, ARG and DR) and other external experts on research methods. Four members of the team will coordinate the SRs (YVM, ARG, CS, and AR). The team of reviewers included professionals with medical background, professionals with methodological background, professionals with both medical and methodological background, professionals with neither medical nor methodological background but involved in the project as study nurse or similar, doctoral, psychology and medical students.

For each SR (i.e. each drug-indication pair), a designated lead reviewer will prepare a topic-specific protocol. This will have the same structure as the PT, but will be adapted appropriately for each drug-indication pair. Furthermore, an unsystematic literature search will be conducted (using PubMed and the Cochrane library) prior to the search in order to identify additional relevant aspects and outcomes to be incorporated in the search and taken into consideration for the risk-to-benefit ratio assessment.

\section{Study inclusion criteria Types of studies}

The SRs will include systematic reviews (including Cochrane reviews), meta-analyses from SRs, controlled interventional studies (e.g. RCTs) and observational studies (i.e. cohort, case-control, cross-sectional, and registry studies).

We will exclude editorials, opinion papers, case reports, case series, narrative reviews, letters, and qualitative studies.

\section{Types of participants}

Studies with a sufficient number of people $\geq 65$ years old will be included. For Cochrane reviews, systematic reviews and meta-analyses:

- If overall mean or median age is $\geq 65$ years old.

- If overall mean or median age $<65$ but a subgroup analysis is provided giving relevant results for people $\geq 65$ years.

- If overall mean or median age is not reported but more than $80 \%$ of the studies report a mean or median age $\geq 65$ years.

For controlled interventional studies and observational studies:

- If $\geq 80 \%$ of participants are $\geq 65$ years.

- If less than $80 \%$ of participants are aged $\geq 65$, but a subgroup analysis is provided giving relevant results for people $\geq 65$ years. 
Studies differ in their age inclusion criteria and may report findings for populations or sub-groups defined by different age thresholds than 65 , e.g. $>70$ years. In such cases we will extract and use the results as reported in the study as long as the subgroup is $\geq 65$ years.

\section{Types of interventions}

Studies will be included if reporting on the efficacy and/ or safety of the target chemical substances or drug classes as monotherapy or in combination with any other drug for the treatment of the target indication or indications versus placebo, no treatment, other drugs or a non-pharmacological intervention. We will exclude studies focusing only on acute/short term conditions (e.g. acute management of heart failure or gastrointestinal bleeding in the emergency room).

\section{Types of outcomes}

We will include any of the following clinically relevant endpoints as primary outcomes for the SRs being conducted:

- Quality of life

- Mortality

- Life expectancy

- Hospitalization

- Cognitive impairment or cognitive status

- Functional impairment or functional status

- Cardiovascular event including stroke

- Renal failure

- Composite end points including any of the above (extraction of individual outcomes will be done if reported by original studies)

- Adverse drug event

- Any of the above evaluated as safety endpoints

Additionally, other relevant outcomes identified for the specific drug classes will be taken into consideration. Outcomes will be extracted in their published data format (e.g. dichotomous, continuous). Due to the fact that we expect a low number of articles to be included for most SRs we will not restrict lengths of follow-up. We will exclude studies evaluating only surrogate endpoints (e.g. blood pressure).

\section{Timing}

No time limit will be used for searches 1 and 2 but a limit of the last 10 years will be used for search 3B.

\section{Setting}

Any setting will be included but it should be on the management of chronic conditions. We will not include studies focused on acute treatment.

\section{Language}

No language restriction will be applied, but we will only include studies that can be read by a member of the research team (English, German, Finnish, Italian and Spanish).

\section{Search method}

The searches will be performed centrally by trained researchers at the University of Manchester (YVM and AW). Our search strategy will have a staged approach where we initially search for systematic reviews and metaanalyses (and stop if an existing review is found, conducted in the previous 2 years, that meets all the objectives of our own review), but if relevant reviews are not found, then search for individual studies (controlled intervention and observational studies). There will be a total of four stages of search, by which each subsequent stage will only be undertaken if the previous one fails to yield high quality results or if the research team decides that the evidence identified so far is not sufficient, or of sufficient quality, to enable an evidence based recommendation to be made.

- Search 1: systematic reviews and meta-analyses in the Cochrane Database of Systematic Reviews (OVID interface, 2005 onwards) and Database of Abstracts or Reviews of Effects (DARE, OVID interface, 1991 onwards).

- Search 2: systematic reviews and meta-analyses in MEDLINE (OVID interface, 1946 onwards), EMBASE (OVID interface, 1974 onwards), Health Technology Assessment (HTA, OVID interface 2001 onwards) and International Pharmaceutical Abstracts (IPA, OVID interface 1970 onwards).

- Search 3A: controlled intervention and observational studies from systematic reviews and meta-analysis not included in searches 1 and 2 but containing eligible studies.

- Search 3B: controlled intervention and observational studies in MEDLINE, EMBASE, HTA and IPA. This search will be limited to the last 10 years.

In addition to database searches, the references of included studies will be checked to obtain a comprehensive list of studies. The citations will be scrutinized and the full manuscripts will be obtained of all citations potentially meeting the inclusion criteria. Study protocols will be also collected to consider for future updates of the SRs. A list of excluded studies after full-text check will be reported for each SR.

The PICOS-framework will be used in the development of the search terms (population, intervention, comparison, outcomes and study design). The following schema for the search terms/MeSH Terms will be used: 
Theme 1: Population: "old" adult"," "old" people", "geriatric patient"", elder", also the MeSH terms "aged" and "frail elderly"

Theme 2: Indication: search terms/MeSH terms for the most frequent chronic conditions related to the target drugs

Theme 3: Drug: search terms/MeSH terms for the target drugs

Theme 4: Outcome: "quality of life", "mortality", "life expectancy", "cardiovascular event", "hospitalisation", "hospitalization", "cognitive impairment", "cognitive status", "functional status", "functional impairment", "renal failure", "adverse drug event", "falls" and also the MeSH terms "renal insufficiency", adverse effects, "drug toxicity", "patient safety", "delirium"

Theme 5: Study: search terms/MeSH terms for study design. For this theme, we will use specific search terms for both systematic reviews and individual studies. For systematic reviews, we have adapted a search filter from PubMed [25]. MEDLINE is the database used by PubMed but we will also use other databases. Therefore, corresponding search terms will be specified for all databases. For individual studies, we will use the search filters suggested in the Cochrane Handbook for randomised trials [23]. For observational studies, we will use the search filters validated by Fraser in 2006 [26]. The search terms used by MEDLINE and EMBASE can vary and Fraser (2006) also includes specific search filters for each of these databases which we will use as recommended. The full list of search terms can be seen in the example in Additional file 3.

In the search, all terms within a theme will be connected by "OR", and the five themes will be connected by "AND". We are using OVID as the search engine to cover all target databases [27].

Additional file 3 shows a complete example of the search done for the SR on metformin for the management of type-2 Diabetes.

\section{Data management}

Literature search results will be uploaded to Endnote X7 reference management software package. Endnote will be used to retrieve search results and to de-duplicate references.

\section{Selection of studies}

Titles and abstracts derived from each search will be assessed by two independent researchers (at least one with a medical background) to identify studies which meet the inclusion criteria. The full manuscripts will be obtained for all titles that appear to meet the inclusion criteria or where there is any uncertainty for inclusion.
The reviewers will resolve any disagreements by discussion and, if necessary, by consulting a third reviewer. At the end of each search stage, based on assessment of the quality and quantity of the evidence obtained so far a decision will be made whether or not to proceed to the next stage of search. The list of references to be included for assessment in Search 3A will be prepared by the researchers in parallel to the study selection for Searches 1 and 2.

\section{Data extraction}

One of the reviewers will independently perform data extraction of the included studies using a standardised and piloted data collection form [see Additional file 4] specific to each type of study design (i.e. systematic reviews, controlled interventional studies or observational studies). The second reviewer will check the form and if in their view it is not completely or accurately filled in, will discuss it with the first reviewer and, if necessary, also consult a third reviewer. Data extracted will include the specific drugs and dosages under study, study methods, time to follow-up, characteristics of the participants (e.g. setting, age, comorbidity, functional status and cognitive status), outcomes and results.

\section{Quality appraisal}

Quality appraisal will be done using validated assessment tools for each type of study design. Systematic reviews and meta-analyses will be assessed using a measurement tool for the assessment of systematic reviews (AMSTAR) $[28,29]$. Clinical trials will be assessed following the Cochrane Collaboration's tool for assessing risk of bias addressing the seven specific domains: sequence generation, allocation concealment, blinding of participants and personnel, blinding of outcome assessment, incomplete outcome data, selective outcome reporting and "other issues" [23]. The domain of "other issues" will be answered using a list of specific domains for different trial designs. We will also use the second part of the Cochrane Collaboration's tool to assign a judgement relating to the risk of bias for each study: "Low", "High", or "Unclear" risk of bias [23]. Observational studies will be assessed using a selection of questions extracted from the Critical Appraisal Skills Programme (CASP) [30, 31].

For included systematic reviews or meta-analyses used to develop recommendations, both overall quality and also the quality of each included study will be assessed, except where quality appraisal has been undertaken and reported by the study's own authors.

\section{Dealing with duplicate and companion publications}

All publications from a primary study will be considered for inclusion. We will make clear which publications 
came from the same study when we report the results of the SRs.

Due to our staged approach, it is quite possible that a publication will be found in a search for individual studies as well as in the previous search for systematic reviews or meta-analyses. Individual studies which are part of an already included systematic review or metaanalysis will be included in their own right if they provide additional relevant evidence that would otherwise be omitted. We will transparently report any such instances with the results of the SRs.

\section{Data synthesis}

Our SRs are focused on people aged 65 or older. Our pilot work and initial scoping of the literature suggested that very few, if any, relevant individual trials or existing systematic reviews have researched or reported exclusively on this group. Hence we anticipate that a significant part of the available evidence will come from subgroup analyses undertaken within studies of much wider age-group populations.

For SRs where we find sufficient numbers of studies that have reported results specific to our target group we will undertake meta-analysis to combine estimates of effect size across these. Clinical heterogeneity (e.g. study differences in populations, interventions and outcomes) will need to be considered and we will only combine effect estimates if (a) the outcome reported is in all essentials the same across the studies; (b) the study designs are the same (e.g. RCTs and observational studies will not be pooled); (c) the included populations, mode of drug delivery and study follow-up times are all reasonably comparable. Statistical heterogeneity will be assessed through calculation of the $I^{2}$ heterogeneity index. However, in all cases we will conduct both a fixed-effects and a random-effects meta-analysis, the latter using the DerSimonian method unless counter indicated [32]. We will not base use of the random-effects model on a statistically significant $I^{2}$ value, as true heterogeneity is often present even when this test is nonsignificant [33].

Funnel plots will be used to look for evidence of publication bias and where the number of studies being combined is ten or more we will apply Egger's test for funnel plot asymmetry [23].

If there is not sufficient data to perform meta-analysis, we will provide a narrative synthesis describing characteristics and findings of included studies. For each SR, we will group studies by specific drugs and indications (if applicable) and by outcomes (which will be further categorised as "risks" and "benefits"). The results for each SR will be summarised in tables for: a) characteristics of included studies (i.e. study design, participants, intervention, comparator, follow-up, and outcomes) and b) summary statistics for each outcome (e.g. odds ratios, relative risks, and hazard ratios). All studies will be included in the narrative synthesis regardless of their quality rating, which will be reported and taken into account when drawing conclusions.

\section{Identification of references of interest for the development of recommendations}

Reviewers will identify additional references which did not fulfil the inclusion criteria of the SRs but which they consider of interest for the development of recommendations. These references may be retrieved either from any of the searches of the SR process or from other sources (i.e. snowballing). References of interest are expected to mostly be studies of younger people but which provide relevant evidence on risks-benefits for a given drug-indication pair; however they may also relate to clinical guidelines or expert consensus. Thus, the identification of these references mostly aims at providing the research team with some evidence on the use of the drugs under study in younger populations.

\section{Development of recommendations}

The studies included and the references considered of interest will be summarised in a document which will be used in team meetings to discuss the recommendations. This document will include: a) relevant information from all selected studies such as study design, target population and sample size, intervention and comparison groups, outcomes, main results, subgroup analysis if applicable; b) results of the quality appraisal of the studies; and c) proposed recommendations to discuss. Initial team meetings will be held by the reviewers of each drug-indication pair, a geriatrician and researcher (ARG) and a senior clinician and researcher (AS). In a later stage the Finish team of editors from Duodecim Medical Publications Ltd. lead by IK will participate and approve the recommendations.

The aim here is to use the overall evidence identified on both risks and benefits of each drug for each indication or indications, to develop recommendations about when not to use or to discontinue the drug or when to reduce its dose. It will be important that this process takes full account of the extent and quality of the identified age-group specific evidence, to avoid overinterpretation and to recognise where the evidence is insufficient to draw any firm conclusions. The following hypothetical situations may happen: 1) the evidence on risks on the use of a medication for older people outweighs the evidence on benefits; in this case a recommendation on the potential discontinuation of the drug will be developed; 2) the evidence on benefits outweighs the evidence on risks; in this case no recommendation on the discontinuation of the drug will be developed; 3) 
the evidence is insufficient on both risks and benefits; in this case we will take into consideration the evidence on general population and clinical guidelines as identified from the additional references of interest and discuss, for each individual case, whether a recommendation on the potential discontinuation of the drug could be developed where clinicians are drawn attention about the lack of evidence on its use for this age group. Recommendations will be given a strength (weak or strong) and the evidence underlying each recommendation will be given a quality (low, moderate or high) following the GRADE methodology [34-36]. The final recommendations will be worded following a standardised schema according to their strength and the quality of their evidence. Recommendations may have to be put into the context of other drugs which are used for the same indication. Data sources, as well as reasons for upgrading or downgrading the evidence will be transparently reported.

Recommendations will be built into the CMR tool and displayed for general practitioners using the tool if their patients are prescribed the relevant drug for the corresponding indications. Recommendations may be linked to certain conditions or symptoms as well, and should allow the process of shared decision making and the reduction of inappropriate prescribing. The standardised schema to word recommendations can be seen in Table 2 .

General practitioners using the CMR tool will be informed and trained that the recommendations made by the tool do not substitute for careful individual clinical considerations or clinical guidelines, but are only intended to support the clinical decision making process.

\section{Discussion}

This protocol has been prepared for a series of systematic reviews which will provide research-based evidence to develop recommendations to reduce inappropriate polypharmacy in older people as part of the CMR tool of the

Table 2 Standardised schema to word recommendations

Strength of the recommendation
- Weak recommendation:
It is suggested to discontinue [include the drug] for the management
of [include the indication] because it [include the wording regarding
the quality of the evidence]
- Strong recommendation
It is recommended to discontinue [include the drug] for the
management of [include the indication] because it [include the
wording regarding the quality of the evidence]
Quality of the evidence
- High quality of evidence: increases/decreases/is associated with ...
- Moderate quality of evidence: appears to increase/decrease/be
associated with ...
- Low quality of evidence: may increase/decrease/be associated
with ...

PRIMA-eDS project. We developed a staged approach methodology for a set of systematic reviews which has the advantage of looking at recently synthesised researchbased evidence in searches 1 and 2 (i.e. systematic reviews and meta-analysis) and, only if necessary, evidence from single studies in searches $3 \mathrm{~A}$ and $3 \mathrm{~B}$ (i.e. individual controlled intervention and observational studies). One purpose of Search 3A is to ensure that relevant individual studies are not missed by being excluded along with a systematic review or meta-analysis - of which they might be part - that failed to meet our inclusion criteria for synthesised evidence.

Full systematic reviews can be time consuming [37] and we are aware of other methods to retrieve researchbased evidence, such as "rapid reviews". However, we decided to tailor our methodology along "traditional" systematic review lines in order to guarantee good quality of the evidence through use of the manuals from the Cochrane collaboration [23] and the PRISMA statement for reporting systematic reviews [24]. Our staged methodology also enables us to reduce the time needed for complete "traditional" systematic reviews thus making it possible to cover a large number of drugs and indications within the limited time frame of our EU-project.

We believe our approach also overcomes some of the limitations of a rapid review, for which there is a lack of published guidelines or explicit methods [37, 38]. Some rapid reviews do not have clear research questions including participants, interventions, comparisons and outcomes (PICOS) [37]. In addition, the streamlining process of rapid reviews can compromise overall quality, for example, to save time a rapid review might not do a comprehensive search in all relevant databases or it might not have more than one reviewer to select, extract data and appraise the quality of included studies [39].

Our search strategy includes specific terms for older people. This might limit our ability to capture all studies that include evidence on older people. However, we will use a broad range of terms for older people (e.g. old* adult*, aged*, elder*, >65) and search for these terms across multiple fields (title, abstract, and subject headings) in order to reduce the risk that important studies may not be picked up.

We have identified 75 drugs or drug classes as the most commonly prescribed to older people. Although it is not possible to review all of these within the PRIMA-eDS European project, more drugs or drug classes will be reviewed after the first 20 SRs are completed under the project. The Comprehensive Medication Review (CMR) tool is based on drug databases by Duodecim Medical Publications Ltd. and the results of the PRIMA-eDS systematic reviews. Most of the seventy-five drugs/drug classes are covered by the CMR tool with recommendations based on other sources or methods. For example, there 
are specific recommendations on older people regarding the use of antidepressants, corticosteroids for asthma, methotrexate, among others.

Older people comprise the great majority of consumers of drugs used in the treatment of chronic diseases, yet there has been little previous work to systematically assess the research evidence for usage in this population. The specific approach we have developed and described in this protocol will allow us to conduct a large number of reviews in a systematic and efficient manner but still adhere to recognised standards of quality. The resulting reviews will represent an important step forward in addressing this major gap in knowledge. Through their incorporation in the PRIMA-eDS CMR tool and through other forms of dissemination, we hope that the recommendations that emerge will be of assistance to both general practitioners and policy-makers in reducing unnecessary polypharmacy and inappropriate prescribing in this vulnerable age-group.

\section{Additional files}

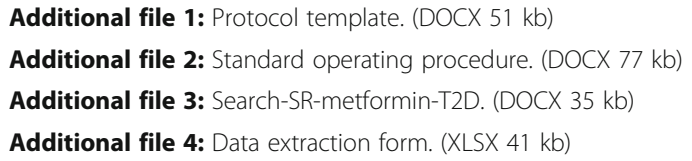

\begin{abstract}
Abbreviations
AMSTAR: Measurement Tool to Assess Systematic Reviews; CASP: Critical Appraisal Skills Programme; CMR: Comprehensive Medication Review; COPD: Chronic obstructive pulmonary disease; DARE: Database of Abstracts or Reviews of Effects; GRADE: Grading of Recommendations Assessment, Development and Evaluation; HTA: Health Technology Assessment; IPA: International Pharmaceutical Abstracts; OsMed: Medicines Utilisation Monitoring Centre [L'Osservatorio Nazionale sull'Impiego dei Medicinali Italian]; PICOS: Population, intervention, comparison, outcomes and study design; PRIMA-eDS: Polypharmacy in chronic diseases: Reduction of Inappropriate Medication and Adverse drug events in elderly populations by electronic Decision Support; PRISMA: Preferred Reporting Items for Systematic Reviews and Meta-Analyses; PT: Protocol template; RCTs: Randomised controlled trials; SOP: Standard Operating Procedures; SR: Systematic review
\end{abstract}

\section{Acknowledgements}

We thank the team of reviewers for their valuable comments during the workshops which contributed to refine the methods of this study. Reviewers: Adrine Woodham, Anna Vögele, Barbara Faller, Charles Adeniji, Germaine Stern-Kuthe, Gisela Schott, Maren Meinshausen, Neha Kaushik, Nik Koneczky, Roman Schlager, Sabine Weißbach, Thekraiat Al Qur'An. Students: Angelika Taetz, Ann-Kathrin Buecherl, Greta Funkhouser, Irene Rothermundt, Moritz Kröger, Philipp Daniels, Raniah El-Jezawi, and Steffen Hübner.

We thank Annette Barber, Anke Kettermann and Dragana Mitric who supported the literature searches of the pilot review.

We thank Dr. Ralph Möhler for his contribution as expert during the workshops.

\section{Funding}

The PRIMA-eDS study was supported by a grant from the European Commission within the 7th Framework Programme (Grant No. 305388-2). The work of YM was also supported by a grant from the NIHR Greater Manchester Primary Care Patient Safety Translational Research Centre.

Publication of this article was funded by the UK National Institute for Health Research School for Primary Care Research, University of Manchester.

\section{Availability of data and materials}

The data supporting the conclusions of this article is included within the article (and its additional files).

\section{About this supplement}

This article has been published as part of BMC Geriatrics Volume 17 Supplement 1 2017: The Evidence Base of Frequently prescribed drugs in older Patients: A series of systematic reviews as a basis for recommendations in the PRIMA-eDS-tool to reduce inappropriate polypharmacy. The full contents of the supplement are available online at https://bmcgeriatr.biomedcentral.com/articles/supplements/ volume-17-supplement-1.

\section{Authors' contributions}

AS, AE, REED and IK conceptualised the first study and prepared the first draft of the protocol template for the systematic reviews (SR). YVM, ARG, DR and AS finalised the protocol template and refined the methods. WM and ARG piloted the protocol template and developed the Standard Operating Procedure and the work documents. CS, AR and IK did significant contributions to refine the methods. WM and ARG drafted the manuscript, supported by DR and AS. All the authors critically reviewed the drafts and approved the final manuscript.

\section{Ethics approval and consent to participate}

Not applicable.

\section{Consent for publication}

Not applicable.

\section{Competing interests}

The authors declare that they have no competing interests.

\section{Publisher's Note}

Springer Nature remains neutral with regard to jurisdictional claims in published maps and institutional affiliations.

\section{Author details}

${ }^{1}$ NIHR School for Primary Care Research, Manchester Academic Health Science Centre, University of Manchester, Manchester, England, UK. ${ }^{2}$ Institute of General Practice and Family Medicine, Witten/Herdecke University, Witten, Germany. ${ }^{3}$ Department of Geriatrics, University Hospital Parc de Salut Mar, Barcelona, Spain. ${ }^{4}$ Family Medicine Unit, Faculty of Medicine, University of Colombo, Colombo, Sri Lanka. ${ }^{5}$ Duodecim Medical Publications Ltd, Helsinki, Finland.

Published: 16 October 2017

\section{References}

1. Hajjar ER, Cafiero AC, Hanlon JT. Polypharmacy in elderly patients. Am J Geriatr Pharmacother. 2007:5(4):345-51.

2. Hovstadius B, Hovstadius K, Astrand B, Petersson G. Increasing polypharmacy an individual-based study of the Swedish population 2005-2008. BMC Clin Pharmacol. 2010;10:16.

3. Marengoni A, Angleman S, Melis R, Mangialasche F, Karp A, Garmen A, Meinow B, Fratiglioni L. Aging with multimorbidity: a systematic review of the literature. Ageing Res Rev. 2011;10(4):430-9.

4. Fortin M, Stewart M, Poitras ME, Almirall J, Maddocks H. A systematic review of prevalence studies on multimorbidity: toward a more uniform methodology. Ann Fam Med. 2012;10(2):142-51.

5. Banerjee A, Mbamalu D, Ebrahimi S, Khan AA, Chan TF. The prevalence of polypharmacy in elderly attenders to an emergency department - a problem with a need for an effective solution. Int J Emerg Med. 2011;4(1):22.

6. Slabaugh SL, Maio V, Templin M, Abouzaid S. Prevalence and risk of polypharmacy among the elderly in an outpatient setting: a retrospective cohort study in the Emilia-Romagna region, Italy. Drugs Aging. 2010;27(12):1019-28.

7. Barat I, Andreasen F, Damsgaard EM. The consumption of drugs by 75-year-old individuals living in their own homes. Eur J Clin Pharmacol. 2000;56(6-7):501-9.

8. Onder G, Liperoti R, Fialova D, Topinkova E, Tosato M, Danese P, Gallo PF, Carpenter I, Finne-Soveri H, Gindin J, et al. Polypharmacy in nursing home in Europe: results from the SHELTER study. J Gerontol A Biol Sci Med Sci. 2012;67(6):698-704. 
9. Van Spall HG, Toren A, Kiss A, Fowler RA. Eligibility criteria of randomized controlled trials published in high-impact general medical journals: a systematic sampling review. JAMA. 2007;297(11):1233-40.

10. Crome P, Lally F, Cherubini A, Oristrell J, Beswick AD, Clarfield AM, Hertogh C, Lesauskaite V, Prada Gl, Szczerbinska K, et al. Exclusion of older people from clinical trials: professional views from nine European countries participating in the PREDICT study. Drugs Aging. 2011;28(8):667-77.

11. Boyd CM, Darer J, Boult C, Fried LP, Boult L, Wu AW. Clinical practice guidelines and quality of care for older patients with multiple comorbid diseases: implications for pay for performance. JAMA. 2005;294(6):716-24.

12. Budnitz DS, Shehab N, Kegler SR, Richards CL. Medication use leading to emergency department visits for adverse drug events in older adults. Ann Intern Med. 2007:147(11):755-65.

13. Schuler J, Duckelmann C, Beindl W, Prinz E, Michalski T, Pichler M. Polypharmacy and inappropriate prescribing in elderly internal-medicine patients in Austria. Wien Klin Wochenschr. 2008;120(23-24):733-41.

14. Chutka DS, Takahashi PY, Hoel RW. Inappropriate medications for elderly patients. Mayo Clin Proc. 2004;79(1):122-39.

15. Kaufmann CP, Tremp R, Hersberger KE, Lampert ML. Inappropriate prescribing: a systematic overview of published assessment tools. Eur J Clin Pharmacol. 2014;70(1):1-11.

16. Gruppo di lavoro OsMed. L'uso dei farmaci in Italia. Rapporto nazionale anno 2011. Roma: Il Pensiero Scientifico Editore; 2012.

17. Koper D, Kamenski G, Flamm M, Bohmdorfer B, Sonnichsen A. Frequency of medication errors in primary care patients with polypharmacy. Fam Pract. 2013;30(3):313-9.

18. Brvar M, Fokter N, Bunc M, Mozina M. The frequency of adverse drug reaction related admissions according to method of detection, admission urgency and medical department specialty. BMC Clin Pharmacol. 2009;9:8.

19. Franceschi M, Scarcelli C, Niro V, Seripa D, Pazienza AM, Pepe G, Colusso AM, Pacilli L, Pilotto A. Prevalence, clinical features and avoidability of adverse drug reactions as cause of admission to a geriatric unit: a prospective study of 1756 patients. Drug Saf. 2008;31(6):545-56.

20. Howard RL, Avery AJ, Slavenburg S, Royal S, Pipe G, Lucassen P, Pirmohamed M. Which drugs cause preventable admissions to hospital? A systematic review. Br J Clin Pharmacol. 2007;63(2):136-47.

21. Ruiter R, Visser LE, Rodenburg EM, Trifiro G, Ziere G, Stricker BH. Adverse drug reaction-related hospitalizations in persons aged 55 years and over: a population-based study in the Netherlands. Drugs Aging. 2012;29(3):225-32.

22. Marengoni A, Rizzuto D, Wang HX, Winblad B, Fratiglioni L. Patterns of chronic multimorbidity in the elderly population. J Am Geriatr Soc. 2009:57(2):225-30.

23. Higgins JPT, Green S, (editors): Cochrane handbook for systematic reviews of interventions. Version 5.1.0 [updated March 2011] The Cochrane Collaboration, 2011. Available from http://handbook-5-1.cochrane.org/. Accessed 12 Aug 2017.

24. Liberati A, Altman DG, Tetzlaff J, Mulrow C, Gotzsche PC, loannidis JP, Clarke M Devereaux PJ, Kleijnen J, Moher D. The PRISMA statement for reporting systematic reviews and meta-analyses of studies that evaluate healthcare interventions: explanation and elaboration. BMJ. 2009;339:b2700.

25. U.S. National Library of Medicine. Search Strategy Used to Create the Systematic Reviews Subset on PubMed [http://www.nlm.nih.gov/bsd/ pubmed_subsets/sysreviews_strategy.html. Accessed 28 Feb 2017].

26. Fraser C, Murray A, Burr J. Identifying observational studies of surgical interventions in MEDLINE and EMBASE. BMC Med Res Methodol. 2006:6:41.

27. Ovid Technologies. Ovid [http://www.ovid.com/site/about.jsp. Accessed 28 Feb 2017].

28. Shea BJ, Grimshaw JM, Wells GA, Boers M, Andersson N, Hamel C, Porter AC, Tugwell P, Moher D, Bouter LM. Development of AMSTAR: a measurement tool to assess the methodological quality of systematic reviews. BMC Med Res Methodol. 2007:7:10.

29. Shea BJ, Hamel C, Wells GA, Bouter LM, Kristjansson E, Grimshaw J, Henry DA, Boers M. AMSTAR is a reliable and valid measurement tool to assess the methodological quality of systematic reviews. J Clin Epidemiol. 2009;62(10):1013-20

30. Critical Appraisal Skills Programme. 12 questions to help you make sense of cohort study [http://media.wix.com/ugd/dded87_5ad0ece77a3f4fc9bcd 3665a7d1fa91f.pdf. Accessed 15 May 2017].

31. Critical Appraisal Skills Programme. 11 questions to help you make sense of case control study [http://media.wix.com/ugd/dded87_afbfc99848f6 4537a53826e1f5b30b5c.pdf. Accessed 15 May 2017].
32. Kontopantelis E, Reeves D. Performance of statistical methods for meta-analysis when true study effects are non-normally distributed: a simulation study. Stat Methods Med Res. 2012;21(4):409-26.

33. Kontopantelis E, Springate DA, Reeves D. A re-analysis of the Cochrane library data: the dangers of unobserved heterogeneity in meta-analyses. PLoS One. 2013;8(7):e69930.

34. Guyatt $G H$, Oxman AD, Kunz R, Falck-Ytter $Y$, Vist GE, Liberati $A$, Schunemann HJ, Group GW. Going from evidence to recommendations. BMJ. 2008;336(7652):1049-51.

35. Guyatt GH, Oxman AD, Kunz R, Vist GE, Falck-Ytter Y, Schunemann HJ, GRADE Working Group. What is "quality of evidence" and why is it important to clinicians? BMJ. 2008;336(7651):995-8.

36. Guyatt GH, Oxman AD, Vist GE, Kunz R, Falck-Ytter Y, Alonso-Coello $P$ Schunemann HJ. GRADE Working Group: an emerging consensus on rating quality of evidence and strength of recommendations. BMJ. 2008:336(7650):924-6.

37. Harker J, Kleijnen J. What is a rapid review? A methodological exploration of rapid reviews in health technology assessments. Int J Evid Based Healthc. 2012;10(4):397-410.

38. Watt A, Cameron A, Sturm L, Lathlean T, Babidge W, Blamey S, Facey K, Hailey D, Norderhaug I, Maddern G. Rapid reviews versus full systematic reviews: an inventory of current methods and practice in health technology assessment. Int J Technol Assess Health Care. 2008;24(2):133-9.

39. Ganann R, Ciliska D, Thomas H. Expediting systematic reviews: methods and implications of rapid reviews. Implement Sci. 2010;5:56.

\section{Submit your next manuscript to BioMed Central and we will help you at every step:}

- We accept pre-submission inquiries

- Our selector tool helps you to find the most relevant journal

- We provide round the clock customer support

- Convenient online submission

- Thorough peer review

- Inclusion in PubMed and all major indexing services

- Maximum visibility for your research

Submit your manuscript at www.biomedcentral.com/submit
) Biomed Central 